\title{
La Personalidad de los Rescatistas: Una Comparación entre Rescatistas Novatos y Experimentados
}

\section{Liberating education of Freire, challenges in face-to-face and virtual modalities}

Recepción del artículo: 23-09-2021 ～Aceptación del artículo: 10-12-2021

\begin{abstract}
Aldana Sol Grinhauz ${ }^{1}$ solgrin@yahoo.com.ar

https://orcid.org/0000-0003-2832-339X

Sergio Héctor Azzara ${ }^{2}$ azzarasergio@gmail.com

https://orcid.org/0000-0003-2310-2256

Susana Celeste Azzollini1,2,3,4 susana1060@yahoo.com.ar

https://orcid.org/0000-0002-3192-5087

1,2Facultad del Ejército, Universidad de la Defensa Nacional, Colegio Militar de la Nación. Centro de Investigaciones en Ciencias Sociales y Humanas para la Defensa (CISOHDEF).

1,2,3Investigadora Independiente CONICET

4 Prof. Adjunta regular a cargo Universidad de Buenos Aires
\end{abstract}

Para referenciar este artículo:

Grinhauz, A. S., Azzara, S. H., y Azzollini, S. C. (2022). La Personalidad de los Rescatistas: Una

Comparación entre Rescatistas Novatos y Experimentados. Revista. ConCiencia EPG, 7(1),

$159-171$

https://doi.org/10.32654/CONCIENCIAEPG.7-

$\underline{1.10}$

Autora corresponsal: Sergio Héctor Azzara, azzarasergio@gmail.com Buenos Aires, Argentina

\section{Resumen}

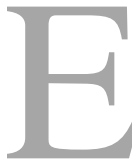

l presente estudio buscó analizar si existen diferencias en rescatistas con más o menos experiencia, en sus dimensiones de personalidad según el modelo del Big Five o Modelo de los Cinco Grandes. Se trabajó con una muestra de 246 rescatistas. El promedio de edad fue de 31,40 años (DT = 10.44). 136 rescatistas eran novatos, y 110 rescatistas tenían experiencia en situaciones de emergencia $\left(M_{\text {años de experiencia }}=\right.$ 9,84; DT = 6.77). Se utilizó una adaptación del instrumento Big Five Inventory. Los rescatistas novatos reportaron una mayor tendencia a la experimentación de sus emociones negativas con mayor intensidad e inestabilidad en comparación con los experimentados. Estos resultados proveen evidencia acerca de la importancia de desarrollar una personalidad resistente para los rescatistas experimentados donde la estabilidad emocional juegue un papel determinante.

Palabras Clave: Personalidad, Rescatistas, Experiencia. 
Abstract

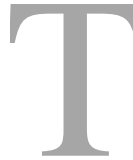

he present study aimed to analyze whether there are differences in rescuers more or less experienced in the five personality factors. We worked with a sample of 246 rescuers. The average age was 31.40 years $(S D=10.44)$. 136 rescatists were novices and 110 rescuers had experience in emergency situations $(\mathrm{M}$ years of experience $=9.84 ; \mathrm{SD}=$ 6.77). An adaptation of the Big Five Inventory instrument was used. Novices rescatists reported a greater tendency to experience their negative emotions with greater intensity and instability compared to experienced ones. These results provide evidence about the importance of developing a resilient personality for experienced rescuers where emotional stability plays a determining role.

Key Words: Personality, Rescuers, Experience.

\section{La Personalidad de los Rescatistas: Una comparación entre rescatistas novatos y experimentados}

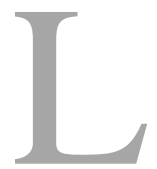

as personas que trabajan en los servicios de emergencia, también llamados rescatistas, eligen conscientemente trabajar en situaciones en las que su propia vida o la de los demás se ven amenazadas. Presencian escenarios como accidentes automovilísticos, incendios, emergencias médicas, la muerte de un integrante del equipo o desastres naturales (Lee et al., 2018; Salters-Pedneault et al., 2010).

Dado el sacrificio que esta profesión implica, ¿por qué algunas personas la eligen? ¿Existen rasgos de personalidad que caracterizan a los rescatistas? Mitchell y Bray (1990) han propuesto que el personal de emergencia elije participar de este tipo de situaciones porque tienen "personalidades muy diferentes de la persona promedio ..." (p. 19). Estos autores sugieren que el personal de emergencia, en general, tienen las siguientes características en común: 1) Muestran una actitud tranquila y mesurada; 2) Suelen aburrirse fácilmente y son extrovertidos; 3) Son más tradicionalistas y conservadores; 4) Son rigurosos con su desempeño, mostrando altos estándares de perfeccionismo en su accionar; 5) Son empáticos, muy dedicados a ayudar a los demás; 6) Están orientados a la acción, aceptan desafíos como comportamientos riesgosos. A este conjunto de características le han otorgado el nombre de personalidad de rescate (Mitchell, 1983).

Una crítica principal a la descripción de la personalidad de rescate de Mitchell y Bray (1990) es la falta de alineación con las teorías de la personalidad más conocidas (Wagner et al., 2009). De acuerdo con esto, un modelo ampliamente investigado $\mathrm{y}$ replicado en distintas partes del mundo es el modelo de personalidad normal del Big Five o Modelo de los Cinco Grandes. Dicho modelo se fundamenta en la hipótesis léxica que postula que la mayoría de las características de la personalidad socialmente relevantes e importantes están codificadas en el lenguaje natural 
(Goldberg, 1993). Estos factores fueron clarificados por Tupes y Christal (1958, 1961) y recibieron el nombre de Big Five por Golberg (1981). Los cinco factores de la personalidad normal son: 1) Extraversion (extraversion), la cual está representada por un enfoque enérgico hacia el mundo social $\mathrm{y}$ material e incluye rasgos como la sociabilidad, la actividad, la asertividad y la emocionalidad positiva; 2) Agradabilidad (agreeableness), supone un conjunto de rasgos caracterizados por una orientación prosocial, altruista y comunitaria hacia los demás; 3) Responsabilidad o escrupulosidad (conscientiousness), la cual se refiere al control de impulsos prescrito socialmente que facilita el comportamiento dirigido a tareas y objetivos, como pensar antes de actuar, retrasar la gratificación, seguir normas y reglas, y planificar, organizar y priorizar tareas; 4) Neuroticismo (neuroticism), factor definido por una emocionalidad negativa que incluye sentirse ansioso, nervioso, triste y tenso; y 5) Apertura a la Experiencia (Openness) implica amplitud, profundidad, originalidad y complejidad de la vida emocional y experimental de un individuo (John et al., 1991; John et al., 2008; John y Srivastava, 1999).

Existen estudios que han evaluado los cinco grandes factores de la personalidad en rescatistas, encontrando que la extraversión y la agradabilidad son los rasgos más característicos de estos trabajadores (Bekkers, 2005; Dudek, 2001; Elshaug y Metzer, 2001; Penner y Finkelstein, 1998; Salters-Pedneault et al., 2010;; Thompson y Solomon, 1991). Por su parte, Flin (2001) han encontrado que la responsabilidad o escrupulosidad constituye un buen predictor de la efectividad del rescatista en situaciones de emergencia mientras que Huang et al. (2019) encontraron que este rasgo se asocia negativamente con el riesgo de contraer trastorno de estrés postraumático (TEPT). Vaulerin et al. (2016) hallaron que el neuroticismo se asocia positivamente con el agotamiento emocional y el cansancio cognitivo; mientras que Wagner et al. (2019) detectaron una asociación entre el mencionado rasgo y la empatía emocional. Por su parte, Fannin y Dabbs (2003), encontraron que una menor apertura a la experiencia y una menor agradabilidad se asocian en forma positiva $y$ estadísticamente significativa con la elección de convertirse en un trabajador de emergencias. En contraste a lo hallado por estos autores, un estudio que se realizó con personal profesional con posibilidades de participación como rescatistas en desastres o emergencias encontró que la apertura a la experiencia se asoció de manera positiva con la efectividad en primera ayuda psicológica (Azzollini et al., 2019).

En resumen, por un lado, se han hallado diferencias de personalidad al comparar rescatistas con la población general $\mathrm{y}$, por el otro, también se han encontrado dichas diferencias entre rescatistas con una mayor eficacia en tareas de rescate respecto de los menos eficaces en tales tareas.

Otro aspecto de relevancia son las diferencias que se pueden encontrar al comparar rescatistas con más años de servicio respecto de los novatos en labores de emergencia. Algunos estudios han respaldado la idea de que una mayor 
experiencia en el trabajo de emergencia ayudaría a proteger contra el efecto de estrés en la salud del trabajador. El papel protector de la experiencia fue avalado por una investigación realizada sobre más de 28,000 trabajadores y voluntarios que han participado en tareas de rescate el 11 de septiembre de 2001 en el World Trade Center (Perrin et al., 2007). La prevalencia del TEPT fue más alta entre los rescatistas que no estaban afiliados a organizaciones específicas y no tenían capacitación y experiencia relevantes. Estos hallazgos sugirieron también que los rescatistas mostraron una mayor capacidad de recuperación al TEPT si se dedicaban a tareas en las que tenían experiencia previa. Otros estudios sugieren que bajo condiciones de presión temporal los rescatistas más experimentados pueden priorizar su atención en las fuentes de estrés y recabar información de valor relevante de su memoria para iniciar acciones eficaces en la actual situación de emergencia basándose en la experiencia pasada (Klein, 1997). La literatura muestra claramente una relación positiva entre experiencia y calidad de la decisión tomada en situaciones de alto nivel de estrés (Reason, 1990).

En suma, existen numerosas investigaciones que han encontrado asociaciones entre la experiencia del rescatista y constructos relevantes para dicha profesión como lo son, la toma de decisiones y la performance en la acción de rescate, la respuesta a situaciones de estrés y el riesgo a desarrollar TEPT, entre otros (Huang et al., 2019; Perrin et al., 2007; Reason, 1990). Sin embargo, son pocos los estudios que profundizan si los rescatistas con más o menos años de servicio, muestran diferencias en sus rasgos de personalidad.

Según investigaciones previas, los rasgos de personalidad y temperamento son moderadamente estables en preescolar y se vuelven cada vez más estables hasta la edad adulta media (Bazana y Stelmack, 2004; Briley y Tucker-Drob, 2014; Roberts y Del Vecchio, 2000). Este hallazgo se ha denominado el principio de continuidad acumulativa del desarrollo de la personalidad (Roberts y Mroczek, 2008). Sin embargo, según indagaciones recientes, los rasgos de personalidad no son inmunes a la experiencia (Caspi et al., 2005). Los estudios en genética del comportamiento han establecido que las diferencias individuales de temperamento $\mathrm{y}$ personalidad, medidas incluso durante los primeros años de vida, son sólo parcialmente heredables y están influenciados por experiencias ambientales (Caspi et al., 2005).

En relación con el presente trabajo, los experimentos naturales permiten a los investigadores examinar los efectos de los choques exógenos, definidos como eventos negativos inesperados o impredecibles (Akee et al., 2018). Grandes eventos colectivos como desastres naturales (Damian et al., 2021) crean situaciones que afectan a muchas personas a la vez. Existe un estudio que analizó el cambio de personalidad después de un desastre natural, específicamente, un terremoto (Milojev et al., 2014). Este estudio analizó los cambios en seis rasgos de personalidad (extraversión, amabilidad, conciencia, estabilidad emocional, apertura a la 
experiencia y honestidad-humildad) antes y después de los terremotos de Christchurch de 2010/2011 en una muestra longitudinal de Nueva Zelanda, de residentes que se vieron afectados o no por los terremotos. Los rasgos de personalidad mostraron una estabilidad notable, con una sola excepción: aquéllos que fueron afectados por los terremotos disminuyeron levemente en estabilidad emocional durante el período de test-retest de 2 años en relación con aquéllos que no se vieron afectados por los terremotos.

Por todo lo planteado, resulta relevante estudiar las diferencias de rasgos de personalidad de los trabajadores de la emergencia en función de su experiencia. Por lo tanto, el objetivo es indagar si existen diferencias en los cinco factores de personalidad entre rescatistas experimentados (con mayor cantidad de eventos ambientales negativos vividos a lo largo del tiempo) y principiantes. La hipótesis que guía este estudio es que existen rasgos de personalidad que van a presentar diferencias significativas cuando se comparen los rescatistas novatos contra los más experimentados.

\section{Método}

Diseño: Se llevó adelante un estudio transversal y comparativo. En resumen, cada sujeto completó en un solo encuentro el Big Five Inventory y la Encuesta sociodemográfica. Posteriormente, se dividió la muestra en dos grupos según su experiencia en la tarea y se compararon las 5 dimensiones de personalidad.
Participantes: La muestra fue no probabilística intencional. Estuvo conformada por 246 rescatistas $(82$ mujeres y 164 varones) de 7 cuarteles de la zona de AMBA (Área Metropolitana y Gran Buenos Aires), una sede de la Cruz Roja del partido de Tigre (San Fernando), una sede de Defensa Civil de Morón, y un grupo de cadetes del Ejército Argentino pertenecientes al Colegio Militar de la Nación que habían colaborado en la inundación de la Ciudad de La Plata. El promedio de edad fue de 31,40 años (DT = 10.44). Los novatos eran todos aquellos rescatistas que, al momento de la investigación, manifestaron no haber tenido experiencia en situaciones de emergencia, es decir, habían ingresado hacía menos de un año al servicio y se encontraban en proceso de capacitación para tomar sus funciones paralelamente al cumplimiento de tareas de rescate que involucraban pocas personas (choque de autos o incendios de poca magnitud entre otra tareas) aunque no habían asistido, hasta el momento, en situaciones de desastre masivo (choque de trenes, incendios de gran magnitud, atentados). Los experimentados eran todos aquellos rescatistas que poseían al menos un año de experiencia en situaciones de emergencia. Del total de rescatistas, 136 eran novatos y 110 tenían experiencia en situaciones de emergencia $(\mathrm{M}$ años de experiencia $=9,84 ; \mathrm{DT}=$ 6.77).

Instrumentos:

Encuesta sociodemográfica en la cual se indagó la edad, el sexo, la cantidad de años de servicio y establecimiento de trabajo. 
Big Five Inventory (BFI; John et al., 1991). La finalidad de este instrumento es la evaluación de los cinco grandes rasgos de la personalidad: 1) agradabilidad; 2) apertura a la experiencia; 3) responsabilidad o escrupulosidad; 4) extraversión y 5) neuroticismo. Posee 44 ítems que se contestan en una escala Likert de 5 opciones que van desde 1 (muy en desacuerdo) a 5 (muy de acuerdo). En este estudio se utiliza la adaptación realizada en Argentina por Castro Solano y Casullo (2001), la cual cuenta con evidencias de validez referida al constructo y estudio de su confiabilidad mediante Alpha de Cronbach, arrojando valores adecuados: Extraversión: .76, Agradabilidad: .71, Responsabilidad: .81, Neuroticismo: .70, Apertura a la Experiencia: .74

Procedimiento: Se pactó un encuentro con las Sedes en las cuales los rescatistas prestaban sus servicios. En dicho encuentro se administró el BFI y la encuesta sociodemográfica. Se garantizó la confidencialidad de los datos obtenidos y los rescatistas aceptaron participar de forma voluntaria. En todos los casos, los participantes firmaron un consentimiento informado.

Análisis de datos: Se categorizó la variable experiencia como dicotómica: novatos y experimentados.

Con relación al análisis de datos, se realizaron análisis estadísticos descriptivos (medias y desviaciones estándar) para todas las variables.

Posteriormente, y para controlar el error tipo I, se calculó un análisis multivariado de la varianza (MANOVA) para examinar diferencias entre experimentados y novatos (variable independiente) en los cinco rasgos de personalidad evaluados a partir del BFI (variable dependiente). Previamente, se corroboró el cumplimiento del supuesto de homocedasticidad mediante el contraste de Levene y la prueba de M-Box que asume que las matrices de covarianza son iguales en todos los grupos (Hair et al., 1999).

Las variables dependientes se hallaron correlacionadas con la matriz de correlación, detectándose resultados estadísticamente significativos en la prueba de esfericidad de Bartlett $\left(X^{2}=263.904 ; 10\right.$ $g l ; p=.000$ ).

Si bien no se cumplió con el supuesto de normalidad, al tener un tamaño muestral lo suficientemente grande, su violación no influye en los resultados de los análisis (Hair et al., 1999). Todos los análisis fueron efectuados con el SPSS (versión 25)

\section{Resultados}

En un principio, se consideraron las medias más elevadas de los rescatistas a nivel general, es decir, sin dividirlos entre novatos y experimentados. En la Tabla 1 se puede observar que la media más elevada fue la de apertura a la experiencia seguida por la agradabilidad. Esto quiere decir que los rescatistas en su totalidad, se autoreportaron como ingeniosos, originales, reflexivos, curiosos, amables y con disposición para ayudar al prójimo desinteresadamente. 
Posteriormente, para contrastar las medias de las puntuaciones del BFI entre rescatistas novatos y experimentados, se realizó un MANOVA (Peña, 2002) que incluyó a la experiencia como factor y los cinco factores de la personalidad como variables dependientes. El modelo resultó estadísticamente significativo encontrándose diferencias significativas entre los vectores medios de ambos grupos (Lambda de Wilks $=.97, F(5,240)=1.49$, $p<0.01, \eta 2=.03$ ). Para determinar en cuáles rasgos diferían los novatos de los experimentados, se realizaron posteriormente pruebas univariadas (Peña,
2002). Los resultados indicaron que los experimentados puntuaron más alto que los novatos en el rasgo agradabilidad $(F(1$, $244)=4.78, p<.01, \eta 2=.019)$, es decir, se identificaron como más amables, altruistas, empáticos y dispuestos a ayudar al prójimo desinteresadamente en comparación con los novatos. Por su parte, los novatos obtuvieron una media más elevada de neuroticismo $(F(1,244)=5.13, p<.01$, $\eta 2=.021$ ), es decir, se reportaron con una mayor tendencia a la experimentación de sus emociones negativas con mayor intensidad e inestabilidad en comparación con los experimentados.

\section{Tabla 1}

Puntuaciones de los factores de personalidad en la muestra total y en rescatistas experimentados y novatos

\begin{tabular}{lcccc}
\hline Factor & $\begin{array}{c}\text { Total } \\
\mathbf{M}(\mathbf{S d})\end{array}$ & $\begin{array}{c}\text { Novatos } \\
\mathbf{M}(\mathbf{S d})\end{array}$ & $\begin{array}{c}\text { Experimentados } \\
\mathbf{M}(\mathbf{S d})\end{array}$ & F \\
\hline Extroversión & $28.51(4.99)$ & $28.23(4.70)$ & $28.83(5.33)$ & .99 \\
Agradabilidad & $35.69(5.10)$ & $35.05(5.30)$ & $36.47(4.75)$ & $2.18^{*}$ \\
Responsabilidad & $35.13(5.55)$ & $34.79(5.70)$ & $35.55(5.36)$ & 1.07 \\
Neuroticismo & $19.71(5.18)$ & $20.38(5.52)$ & $18.88(4.61)$ & $2.26^{*}$ \\
Apertura & $37.88(5.71)$ & $37.38(6.31)$ & $38.49(4.83)$ & 1.51 \\
\hline
\end{tabular}

$\mathrm{M}=$ media, $\mathrm{SD}=$ desvío estándar, * $\mathrm{p}<.05$

\section{Discusión}

El objetivo del presente trabajo consistió en analizar las diferencias en los cinco factores de la personalidad de acuerdo al modelo de Goldberg (1981) entre rescatistas experimentados $y$ rescatistas novatos.

Los resultados mostraron que los rescatistas experimentados poseen un menor neuroticismo. La estabilidad emocional es claramente necesaria para los rescatistas debido a que están expuestos a situaciones extremas y amenazantes todos los días. Estos hallazgos son compatibles con la personalidad del rescatista postulada por Mitchell (1983) en la cual se considera que una personalidad mesurada y tranquila es una de las características prototípicas de los trabajadores de la emergencia. Sin embargo, el resultado se contrapone al trabajo de Milojev et al. (2014), según el cual aquellos residentes que fueron 
afectados por los terremotos disminuyeron levemente en estabilidad emocional en relación con aquéllos que no se vieron afectados por los terremotos. De acuerdo con estudios previos (Heinrichs et al., 2005; Jeque, 2003; Pajonk et al., 2010), hay ciertos rasgos de personalidad que parecen ser factores de riesgo para desarrollar TEPT y otros trastornos como la depresión o el agotamiento. Por ejemplo, Hui et al. (2001) hallaron que los rescatistas militares que tenían un neuroticismo elevado, reportaron mayores niveles de sintomatología compatible con un TEPT. Consideramos, entonces, que un menor neuroticismo por parte de los experimentados se consideraría necesario para poder permanecer en el servicio. Al tratarse de un estudio transversal, no podemos afirmar que los novatos, con el correr del tiempo, van disminuyendo el neuroticismo y van adquiriendo el temple necesario para hacer frente a situaciones extremas. Por tal motivo, consideramos de relevancia realizar, en futuras líneas de investigación, un estudio longitudinal para observar cómo va comportándose este rasgo en el transcurrir de los distintos años de servicio. Esto permitiría responder un interrogante que ha surgido en el desarrollo del presente estudio acerca de si las personas que tienen mayor estabilidad emocional eligen esta profesión de rescatista, o si es la profesión la que moldea este rasgo con la experiencia. Al respecto, los autores hipotetizamos que la estabilidad emocional se va incrementando con la experiencia debido a que los rescatistas más experimentados deben desarrollar una coraza protectora que favorezca la retención, protección y construcción de recursos frente al estrés cotidiano de sus tareas, De acuerdo a la teoría de conservación de los recursos (COR; Hobfoll, 1989, 2001) las personas tienen un número limitado de recursos y la exposición continua al estrés puede agotar estos recursos limitados. En este sentido, aquellos rasgos de personalidad que provean al rescatista un mecanismo protector para su estabilidad emocional deberán ser desarrollados para permanecer en el servicio. Por otra parte, los rescatistas experimentados mostraron una mayor agradabilidad que los novatos. Estos resultados se corresponden con el estudio de Fannin y Dabbs (2003) quienes hallaron que una menor agradabilidad se asociaba en forma positiva $y$ estadísticamente significativa con la elección de convertirse en un trabajador de la emergencia. Si bien hace falta realizar un estudio longitudinal para poder arribar a conclusiones fehacientes, se puede hipotetizar que la agradabilidad no se trata de un rasgo característico de los rescatistas ingresantes, pero al prestar años de servicio en situaciones de emergencia, es un rasgo que empieza a tomar protagonismo y que, incluso, de acuerdo a estudios previos es característico de este tipo de trabajadores (Bekkers, 2005; Dudek, 2001; Elshaug y Metzer, 2001; Finkelstein, 1998; Penner y Thompson \& Solomon, 1991; SaltersPedneault et al.,2010).

Estos resultados compatibilizan con el concepto de personalidad resistente (Khoshaba \& Maddi, 1999; Maddi, 2006; Maddi et al., 1999), la cual se refiere a una personalidad que combina tres actitudes ante eventos estresantes: compromiso, control y desafío. El compromiso es la voluntad de involucrarse con personas, cosas y situaciones en lugar de estar 
desconectado, aislado o alienado. El control implica luchar para estar a cargo de los eventos que tienen lugar en nuestra vida, a través de nuestra propia capacidad para elegir entre opciones, en lugar de paralizarse en la pasividad y el desamparo. El desafío implica estar dispuesto a aprender constantemente de las propias experiencias en lugar de evitar incertidumbres y amenazas potenciales (Maddi, 2006; Maddi et al., 1999). Estas tres características definen un tipo de personalidad que permite transformar las circunstancias estresantes potencialmente amenazadoras en oportunidades para el crecimiento personal (Maddi, 2002). En este sentido, la muestra de rescatistas experimentados del presente estudio tendría un patrón de personalidad resistente (Khoshaba \& Maddi, 1999), al mostrar un mayor compromiso por el otro (alta agradabilidad) y un mayor control y desafío ante la adversidad lo cual se evidencia en un menor neuroticismo reportado. De hecho, al entrenar a los rescatistas para brindar primera ayuda psicológica a los afectados por un desastre o emergencia se utiliza un protocolo que hace hincapié en dichas características (Azzollini et al., 2017).

Con relación a las limitaciones del presente estudio, como se mencionó anteriormente, al tratarse de un estudio transversal no puede hacerse ninguna inferencia de causalidad entre las variables estudiadas. Es decir, no puede afirmarse que los años de experiencia en rescatismo podrían haber sido un factor determinante en estas diferencias de personalidad encontradas. Será necesario la realización de estudios o experimentos naturales longitudinales para obtener evidencia fehaciente al respecto. Asimismo, el muestreo realizado fue intencional simple, debido a las posibilidades de acceso a los datos. Reunir una muestra de rescatistas fue una ardua tarea dada la dificultad para disponer del tiempo de estos trabajadores que estaban a merced de las emergencias que podían suscitarse. Con respecto a los instrumentos, tanto la encuesta sociodemográfica como el BFI se caracterizaron por la modalidad de autorreporte, lo cual podría repercutir en potenciales distorsiones en las respuestas.

Por otra parte, debe mencionarse que la validez externa del presente estudio se encuentra limitada, ya que faltaron rescatistas de otros rubros como los policías, enfermeros o médicos, entre otros, lo cual podría repercutir en la generalización de los resultados. La mayoría de los participantes, con excepción de los cadetes del Ejército Argentino, trabajaban, al momento del estudio, de forma voluntaria, sin recibir incentivo monetario alguno. Consideramos que, para futuras investigaciones, sería oportuno indagar acerca de las características de personalidad de rescatistas homologando esta última cuestión e incluyendo a otro tipo de trabajadores de la emergencia.

Pese a las limitaciones mencionadas, el presente trabajo echa luz sobre un grupo de trabajadores poco estudiados en Argentina. Estos resultados otorgan valiosa información para la confección del perfil de quienes se ocupan de llevar adelante una de las tareas más difíciles como lo es trabajar en situaciones tan críticas. 


\section{Referencias}

Akee, R., Copeland, W., Costello, E. J., \& Simeonova, E. (2018). How does household income affect child personality traits and behaviors? The American Economic Review, 108(3), 775-827. https://doi.org/10.1257/aer.2016 0133

Azzollini, S. C., Azzara, S. H., Depaula, P. D., Cosentino, A. C., \& Grinhauz, A. S. (2017). Modificación del Protocolo de primera ayuda psicológica en situaciones de crisis.

Azzollini, S. C., Cosentino, A. C., \& Depaula, P. D. (2019). Primera ayuda psicológica para personas afectadas por desastres: Un estudio experimental con personal profesional. Manuscrito en preparación

Briley, D. A., \& Tucker-Drob, E. M. (2014). Genetic and environmental continuity in personality development: A meta-analysis. Psychological Bulletin, 140, 13031331.

http://dx.doi.org/10.1037/a00370 91

Bazana, P. G., \& Stelmack, R. M. (2004). Stability of personality across the life span: A meta-analysis. In $\mathrm{R}$. Stelmack (Ed.), On the psychobiology of personality (pp. 113-144). New York, NY:

Elsevier. http://doi.org/10.1016/B978008044209-9/50009-9
Bekkers, R. (2005). Participation in voluntary associations: Relations with resources, personality, and political values. Political psychology, 26(3),

439-454 https://dx.doi.org/10.1111/j.14679221.2005.00425.x

Caspi, A., Roberts, B. W., \& Shiner, R. L. (2005). Personality development: Stability and change. Annu. Rev. Psychol., $\quad$ 56, 453-484. https://dx.doi.org/10.1146/annur ev.psych.55.090902.141913

Castro Solano, A. \& Casullo, M. M. (2001). Rasgos de personalidad, bienestar psicológico y rendimiento académico en adolescentes argentinos. Interdisciplinaria, 18(1) 65-85.

Damian, R. I., Serrano, S., \& Hill, P. L. (2021). Hurricane exposure and personality development. Journal of Personality, $89(1)$ 35-49. https://doi.org/10.1111/jopy.1254 2

Dudek, B., \& Wichrowski, A. (2001). Application of the big five model for the purpose of selection in fire services. Przeglad Psychologiczny, 44(4), 495-508

Elshaug, C., \& Metzer, J. (2001). Personality attributes of volunteers and paid workers engaged in similar occupational tasks. The Journal of social psychology, 141(6), 752-763. 
http://doi.org/10.1080/00224540 109600586

Fannin, N., \& Dabbs Jr, J. M. (2003). Testosterone and the work of firefighters: Fighting fires and delivering medical care. Journal of Research in Personality, 37(2), 107115.

https://doi.org/10.1016/S00926566(02)00533-0

Flin, R. (2001). Selecting the right stuff: Personality and high-reliability occupations. In B. W. Roberts \& R. Hogan (Eds.), Personality psychology in the workplace (pp. 253-275). American Psychological Association.

https://doi.org/10.1037/10434010

Goldberg, L. R. (1981). Language and individual differences: The search for universals in personality lexicons. Journal of Personality and Social Psychology, 59, 1216-1229.

Hair, J. F., Anderson, R. E., Tatham, R. L., \& Black, W. C. (1999). Análisis multivariante. Prentice Hall.

Heinrichs M., Wagner, D., Schoch, W., Soravia, L. M., Hellhammer, D. H., \& Ehlert, U. (2005). Predicting Posttraumatic Stress Symptons from Pretraumatic Risk Factors: A 2-Year Prospective Follow-Up Study in Firefighters. American Journal of Psychiatry, 162(12), 2276-2286.

https://doi.org/10.1176/appi.ajp.1 62.12 .2276
Hobfoll, S. E. (1988). Theecologyof stress. Hemisphere.

Hobfoll,

S.

E.

(1989).

Conservationofresources: A new attempt at conceptualizing stress. American Psychologist, 44, 513524.

Hobfoll, S. E. (2001). The influence of culture, community, and the nested self in the stress process: Advancing conservation of resources theory. Applied Psychology: An International Review, 50, 337-370.

Huang, J., Wang, X., Li, W., \& An, Y. (2019). The relationship between conscientiousness and posttraumatic stress disorder among young Chinese firefighters: The mediating effect of perceived social support. Psychiatry research, 273 , 450-455. https://doi.org/10.1016/j.psychre s.2019.01.053

Hui, W., Shi, W., Xu, D., Wang, J., Wu, W., \& Men, K. (2001). Influencing factors of posttraumatic stress disorder in the shipwreck related military rescuers in Yantai. Chinese Mental Health Journal, 15, 148-149.

John, O. P., Donahue, E. M., \& Kentle, R. L. (1991). The Big Five InventoryVersions 4a and 54. Berkeley, CA: University of California, Berkeley, Institute of Personality and Social Research.

John, O. P., Naumann, L. P., \& Soto, C. J. (2008). Paradigm shift to the integrative BigFive trait taxonomy: 
History, measurement, and conceptual issues. In O. P. John, R. W. Robins, \& L. A. Pervin (Eds.), Handbook of personality: Theory and research (3rd ed., pp. 114-158). New York, NY: Guilford Press.

Klein, G. (1997). Developing expertise in decision making. Thinking \& Reasoning, 3(4), 337-352.

Khoshaba, D. M., \& Maddi, S. R. (1999). Early experiences in hardiness development. Consulting psychology Journal: practice and research, 51(2), 106.

Lee, J. Y., Kim, S. Y., Bae, K. Y., Kim, J. M., Shin, I. S., Yoon, J. S., \& Kim, S. W. (2018). The association of gratitude with perceived stress and burnout among male firefighters in Korea. Personality and Individual Differences, 123, 205-208.

Maddi, S. R. (2006). Hardiness: The courage to grow from stresses. Journal of Positive Psychology, 1(3). https://doi.org/10.1080/1743976 0600619609

Maddi, S. R., Khoshaba, D. M., \& Pammenter, A. (1999). The hardy organization: Success by turning change to advantage. Consulting Psychology Journal: Practice and Research, 51(2). https://doi.org/117.10.1037//106 1-4087.51.2.117

Milojev, P., Osborne, D., Greaves, L. M., Bulbulia, J., Wilson, M. S., Davies, C. L., ... \& Sibley, C. G. (2014). Rightwing authoritarianism and social dominance orientation predict different moral signatures. Social Justice Research, 27(2), 149-174. https://doi.org/10.1177/1948550 614528545

Mitchell, J. T. (1983). When Disaster Strikes. The Critical Incident Stress Debriefing Process. JEMS: a journal of emergency medical services, 8(1), 36-39.

Mitchell, J. \& Bray, G. (1990). Emergency service stress. Guidelines for preserving the health and careers of emergency service personnel. Prentice Hall.

Pajonk, F. G., Bartels, H. H., Biberthaler, P., Bregenzer, T., \& Moecke, H. (2001). Psychiatric emergencies in preclinical emergency service; incidence, treatment and evaluation by emergency physicians and staff. Der Nervenarzt, 72(9), 685-692.

Penner, L. A., \& Finkelstein, M. A. (1998). Dispositional and structural determinants of volunteerism. Journal of personality and social psychology, 74(2), 525.

Peña, Daniel. (2002). Análisis de Datos Multivariante. Mc Graw-Hill. España.

Perrin, M. A., DiGrande, L., Wheeler, K., Thorpe, L., Farfel, M., \& Brackbill, R. (2007). Differences in PTSD prevalence and associated risk factors among World Trade Center disaster rescue and recovery workers. American Journal of Psychiatry, 164(9), 1385-1394. 
https://doi.org/10.1176/appi.ajp.2 007.06101645

Reason, J. (1990). Human error. Cambridge university press.

Roberts, B. W., \& DelVecchio, W. F. (2000). The rank-order consistency of personality traits from childhood to old age: A quantitative review of longitudinal studies. Psychological Bulletin, 126, 3-25. http://dx.doi.org/10.1037/00332909.126.1.3

Roberts, B. W., \& Mroczek, D. (2008). Personality trait change in adulthood. Current Directions in Psychological Science, 17, 31-35. http://dx.doi.org/10.1111/j.14678721.2008.00543.x

Tupes, E. C., \& Christal, R. C. (1958). Stability of personality trait rating factors obtained under diverse conditions (Vol. 58). Personnel Laboratory, Wright Air Development Center, Air Research and Development Command, United States Air Force.

Salters-Pedneault, K., Ruef, A. M., \& Orr, S. P. (2010). Personality and psychophysiological profiles of police officer and firefighter recruits. Personality and Individual Differences, 49(3), 210-215. https://doi.org/1010.1016/j.paid.2 010.03.037

Thompson, J. \& Solomon, M. (1991). Body recovery teams at disasters:
Trauma or challenge? Anxiety Research, 4, 235-244. https://doi.org/10.1080/0891777 9108248777

Tupes, E. C., \& Christal, R. E. (1961). Recurrent personality factors based on trait ratings (USAF ASD Technical Report No. 61-97). Air Force.

Vaulerin, J., Colson, S. S., Emile, M., ScoffierMériaux, S., \& d'Arripe-Longueville, F. (2016). The big five personality traits and French firefighter burnout: the mediating role of achievement goals. Journal of occupational and environmental medicine, 58(4), e128-e132.

Wagner, S. L., McFee, J. A., \& Martin, C. A. (2009). Effects of traumatic stress on firefighters' world assumptions. Traumatology, 15(1), 75-84. https://doi.org/10.1177/1534765 608323441

Wagner, S. L., Pasca, R., \& Regehr, C. (2019). Firefighters and empathy: does it hurt to care too much? Journal of loss and trauma, 24(3), 238-250. https://doi.org/10.1080/1532502 4.2018.1525035. 